\title{
Deposition and characterization of lines printed through laser-induced forward transfer
}

A. Palla-Papavlu, C. Córdoba, A. Patrascioiu, J. M. Fernández-Pradas, J. L. Morenza, P. Serra*

Departament de Física Aplicada i Optica, Universitat de Barcelona (UB), Martí i Franquès 1, E-08028, Barcelona, Spain

*Corresponding author. Tel.: +34 934039 216; Fax: +34 934039219.

E-mail address: pserra@ub.edu.

\begin{abstract}
The possibility of printing two-dimensional micro patterns of biomolecule solutions is of great interest in many fields of research in biomedicine, from cell-growth and development studies, to the investigation of the mechanisms of communication between cells. Although laser-induced forward transfer (LIFT) has been extensively used to print micrometric droplets of biological solutions, the fabrication of complex patterns depends on the feasibility of the technique to print micron-sized lines of aqueous solutions.

In this study we investigate such a possibility through the analysis of the influence of droplet spacing of a water and glycerol solution on the morphology of the features printed by LIFT. We prove that it is indeed possible to print long and uniform continuous lines by controlling the overlap between adjacent droplets. We show how depending on droplet spacing, several printed morphologies are generated, and we offer, in addition, a simple explanation of the observed behavior based on the jetting dynamics characteristic of the LIFT of liquids.
\end{abstract}

Keywords: LIFT, biopatterning, lines printing, aqueous solutions

\section{Introduction}

In recent years, the topic of micropatterning has received significant attention in the fields of medicine, microbiology, and biotechnology. In particular, small two dimensional architectures of biomolecules play an important role in understanding fundamental biological processes such as cellular growth, intercellular organization, or mechanisms of communication between cells [1]. In addition, these patterns find interesting applications in the isolation and culturing of microbial strains [2]. 
There are various techniques to fabricate small-scale patterns from different types of materials, such as inkjet printing [3, 4] or dip-pen lithography [5]. However, these techniques present some disadvantages, e.g. in the case of inkjet printing the presence of a nozzle, which gives rise to clogging issues, and limits its performance [3], or the slow speed in the case of dip-pen lithography [6].

As an alternative to conventional printing techniques, laser-induced forward transfer (LIFT) has been demonstrated to be feasible for printing biomolecules [7-13], biomolecule structures [14], as well as cells [15], and micro-organisms [16, 17]. In LIFT, a laser beam is focused or imaged through a transparent support onto the backside of a metallic or polymer thin film [11, 14, 16] coated with the material to be transferred. Each pulse promotes the transfer of a small fraction of the thin film material (donor film) onto a receiver substrate that is usually placed parallel and facing the thin film at a short distance (between a few tens and several hundreds of $\mu \mathrm{m}$ ). A pattern of the transferred material can be "written" on the substrate with multiple shots by translating the substrate and the thin film with respect to the laser beam.

The feasibility of LIFT to print patterns of individual droplets has been extensively demonstrated [8, 9, 12-18], and in addition, the mechanisms responsible for droplet formation have been widely investigated by time resolved imaging studies [19-23]. Moreover, the next step forward with respect to droplet deposition is the printing of defect-free continuous, uniform, and stable lines.

Although line printing through LIFT has proved to be feasible for conductive inks [24, 25, 26], these liquids have been precisely engineered for optimum line printing, having rheologies completely different from biomolecule solutions, which are mostly water based solutions. Therefore, we cannot directly extrapolate the results corresponding to conductive inks to the case of aqueous solutions. Furthermore, a systematic study relating the morphological properties of lines printed through LIFT with the main process parameters is currently lacking.

In this work, we investigate the possibility of printing lines of aqueous solutions through LIFT by analyzing the influence of the droplet spacing on the morphology of the laser transferred features. In addition, we provide a possible explanation for the observed dependence of the printed morphologies on droplet spacing which is based on the jetting dynamics characteristic of the LIFT of liquids. 


\section{Experimental details}

The LIFT experimental setup has been described in detail elsewhere [27]. Briefly, the laser pulses generated by a diode pumped Yb:YKW laser system (Amplitude Systems, s-Pulse, 1027 nm wavelength, 450 fs pulse duration) are focused through a microscope objective (50x, NA 0.55 , WD $13 \mathrm{~mm}$ ) onto the backside of the donor film. The donor film is a water and glycerol solution ( $50 \% \mathrm{v} / \mathrm{v}$ ) blade coated ( $15 \mu \mathrm{m}$ thick, estimated by weight measurement) onto a Ti film (50 $\mathrm{nm}$ thickness) coated glass microscope slide. In order to improve wettability, $2 \mathrm{mg} / \mathrm{ml}$ sodium dodecyl sulphate (SDS) surfactant are added to the final solution. The surface tension of the resulting solution is $30 \mathrm{mN} / \mathrm{m}$, and the viscosity $6.5 \mathrm{mPa} \cdot \mathrm{s}$.

Commercially available polysine microscope slides (Gerhard Menzel $\mathrm{GmbH}$ ) are used as receiving substrates. The donor and the receiver substrates are placed at a distance of $100 \mu \mathrm{m}$ through polyimide spacers, and the donor-receiver system placed on a motorized translation stage. The computer controlled stage allows the creation of different features, i.e. from an array of droplets to lines simply by varying the distance between the droplets. The morphology of the generated features was analyzed by an optical microscope coupled with a digital camera (CarlZeiss ${ }^{\mathrm{TM}}$ Axio Imager microscope, equipped with a ProgRes ${ }^{\mathrm{TM}}$ C10 Plus camera). All transfer experiments were carried out in air, at ambient temperature.

\section{Results}

As a result of printing droplet patterns over a variety of droplet spacings, different morphologies emerge. In Fig. 1a an optical microscopy image is shown of printed droplets at different droplet spacings (the droplet spacing decreases from left to right). Droplet spacing is denoted as $\Delta \mathrm{x}$ and is defined as the center-to-center distance between two successive printed droplets, and the droplet overlap is defined as the ratio of overlapped length between two neighboring droplets to the individual droplet diameter on the substrate, as reported in Ref. 28.

Several features can be distinguished in Fig. 1a. At droplet spacings larger than $120 \mu \mathrm{m}$, isolated droplets are transferred onto the substrate. By decreasing droplet spacing to $\Delta \mathrm{x}=100$ $\mu \mathrm{m}$, isolated droplets and sporadic pairs (merging of neighboring droplets) can be noticed. For $\Delta x=80$ and $70 \mu \mathrm{m}$ broken lines form on the substrate. For $\Delta x=60 \mu \mathrm{m}$ a continuous line is formed, which presents a scalloped pattern. Further decreasing the droplet spacing to $\Delta x=50 \mu \mathrm{m}$ eliminates the scalloping and leads to a quite uniform and straight line. However, this result is improved by decreasing $\Delta \mathrm{x}$ to $40 \mu \mathrm{m}$, where the optimum printing spacing is found: at this spacing the printed lines present excellent uniformity. Furthermore, this uniformity can be 
maintained over long distances, as shown in Fig. 1b (the length of the depicted line is $>2 \mathrm{~mm}$ ). Printing droplets even closer together $(\Delta x=30 \mu \mathrm{m})$ leads to the appearance of line bulging, with the bulges connected by regions of uniform and narrower lines. At the smallest analyzed spacing, $\Delta x=20 \mu \mathrm{m}$, splashed features are printed on the substrate. Finally, we should point out that the isolated droplets have a diameter $\left(\mathrm{d}_{0}\right)$ of around $80 \pm 5 \mu \mathrm{m}$, and that the width of the resulting printed features presents always about the same value, except for the lines that feature bulges and splashing.

\section{Discussion}

The observed morphologies (pairs; broken, scalloped, uniform and bulging lines) are not specific to LIFT, but have also been found in inkjet printing experiments [4, 28-33], where the dependence of the onset of each particular morphology on drop spacing has been usually explained in terms of the dynamics of impact of a falling droplet onto the substrate. However, we found that the dynamics of droplet deposition in LIFT is significantly different from that of inkjet printing, which makes it necessary to provide a new interpretation based on the evidence available on the time evolution of the liquid being printed through LIFT [22].

Time-resolved imaging of a LIFT event resulting in the deposition of an isolated sessile droplet of the same solution as that used in our line printing experiments revealed that liquid ejection is mediated by the formation of a needle-like jet which propagates along the gap between donor and receiving substrates [22]. The contact of the jet with the receiving substrate initiates a process which evolves through three clearly differentiated stages, each corresponding to a different time scale, and whose final outcome is a stationary sessile droplet (Fig. 2). During about the first $10 \mu$ s after contact (stage S1), the depositing feature suffers a sudden spreading up to a diameter around $60 \%$ of the final diameter of the sessile droplet. In the second stage (S2), which lasts a few hundreds of $\mu$ s, the jet gently feeds the growing droplet at constant diameter, until the jet breakup terminates the liquid input. In the third stage (S3), lasting a few ms, the previously formed sessile droplet slowly expands at constant volume.

In order to interpret the results of our line printing experiment, we assume that the dynamics taking place are essentially the same as those described in the preceding paragraph. Despite the experimental conditions not being exactly the same as those of Ref. 22, this assumption seems reasonable since the morphology and dimensions of the printed droplets are similar in both cases and it is well known that such jetting dynamics is quite ubiquitous to LIFT experiments leading to the deposition of circular droplets. According to this, we have represented in Fig. 3 top views of all the possible instances of jet impingement depending on drop spacing. For each 
instance, the feature on the left represents the end of the line which is being printed, and on the right it is sketched the perimeter (solid circle, final diameter $\mathrm{d}_{0}$ ) that the next droplet would attain at the end of stage S3 if it were deposited at the corresponding spacing in the absence of the printed line; furthermore, we have also represented the perimeter of the printed feature at the end of stage S1, that delimits the area on which liquid input proceeds during stage S2 (dashed circle, diameter $\mathrm{d}_{1}$ ). All the sketches are to scale.

The sketches of Fig. 3 correlate well with the morphologies in Fig. 1a. The spacings leading to the deposition of isolated droplets $(\Delta x=120,100 \mu \mathrm{m})$ correspond to instances with no overlap between the depicted features (Figs. 3a, b). Broken lines $(\Delta x=80,70 \mu \mathrm{m})$ are obtained when there is overlap between the features, but not between the printed line and the perimeter corresponding to stage S2 (Figs. 3c, d). On the other hand, whenever continuous lines $(\Delta x=50$, $40,30 \mu \mathrm{m}$ ) are obtained, either regular or bulged, there is considerable overlap between the end of the line and the perimeter corresponding to S2 (Figs. 3f, g, h). The scalloped line ( $\Delta x=60$ $\mu \mathrm{m})$, which sets the transition between broken and continuous lines, precisely corresponds to the instance where the perimeter of the line just contacts that of S2 (Fig. 3e). Finally, splashing $(\Delta x=20 \mu \mathrm{m})$ corresponds to a particular instance in which practically the whole perimeter of S2 falls within the printed line (Fig. 3i).

In all the instances shown in Figs 3a-d liquid input takes place when there is absolutely no contact between the droplet being deposited and the already printed feature (line or droplet depending on spacing). Thus, the final outcome of the process will mainly depend on how much the printed droplet spreads during stage S3. At the largest spacing $(\Delta x=120 \mu \mathrm{m})$ there is no overlap between the contact lines of adjacent droplets and, thus, isolated droplets are obtained. The same applies at $\Delta \mathrm{x}=100 \mu \mathrm{m}$, but in this case exceptional spreads eventually allow sporadic contacts, leading to droplet coalescence, which results in the formation of pairs. Such exceptional spreads can be attributed either to occasional over intense laser pulses, or to the pinning of the contact line of the previously printed droplet due to random inhomogeneities of the substrate (as suggested in Fig. 2b, during stage S3 the droplet can spread up to diameters larger than the final $d_{0}$ ). Coalescence occurs due to the capillary flow from the bulk of a liquid feature (droplet or line) towards the overlap region, the flow resulting from the pressure gradient originated by the concavity in that region. But, in addition to coalescence, such flow can have another effect: the unpinning and recoil of the contact line in the printing front as a consequence of liquid depletion around it, and this effect is responsible for obtaining broken instead of continuous lines at the largest spacings allowing overlap $(\Delta x=80,70 \mu \mathrm{m})$. Furthermore, the intensity of the capillary flow must decrease with overlap due to the decrease of concavity. Thus, the recoil of the contact line must become less pronounced, until at a spacing of $60 \mu \mathrm{m}$ 
being so small, that a continuous line is printed, with its scalloped contour clearly evidencing the weakness of the flow: some pinning, at least partial, of the lateral contact line is apparent. At spacings shorter than $60 \mu \mathrm{m}$, however, and according to the instances sketched in Figs. 3f-i, a much stronger flow comes into play. In these cases, with the perimeter of stage S2 overlapping the printed line, the printed line is directly fed by the jet responsible for the formation of the subsequent droplet. Since the duration of stage S2 is at least one order of magnitude shorter than that of S3, we can expect that the flow of the jet feeding the already printed line will be much stronger than the capillary flow. In this new situation, the flow can be strong enough to prevent the pinning of the lateral contact line responsible for the scalloped morphology, which can result in the formation of uniform lines. The onset of bulges at short spacings $(\Delta x=30 \mu m)$ is a consequence of the development of an instability in already formed lines with a high liquid content per unit length, a phenomenon that has been described in detail already [29-31, 34]. Briefly, if any instability generates a minimum bulge somewhere not far from the front of the printed line, such bulge will tend to grow due to the backward capillary flow arising from the pressure gradient between the front and the bulge; the growth will proceed until the length of the bridge connecting bulge and front will be large enough to prevent flow through viscous forces. Finally, in the case of the shortest spacing of $20 \mu \mathrm{m}$, there is so much overlap that the incoming jet tip impinges well onto the previously printed feature, in a region with a considerably large thickness. Thus, the splashing is probably produced by the interaction of such fast jet colliding with the printed liquid.

\section{Conclusion}

The study of the influence of droplet spacing on the morphology of the features obtained through LIFT of a water and glycerol solution has revealed that it is possible to print long and uniform continuous lines by controlling the overlap between adjacent droplets. Depending on the degree of overlap, morphologies apart from uniform lines are obtained: from isolated droplets to splashing, through broken, scalloped, and bulging lines. We have also provided a possible explanation for the different morphologies based on the jetting dynamics of liquids printed through LIFT. According to this explanation, the obtaining of continuous uniform lines would require that the droplet spacing was such that the emitted jet impinges on the line which is being printed at a close distance from the front line. 


\section{Acknowledgment}

This work is funded by MCI of the Spanish Government (Projects MAT2010-15905 and CSD2008-00023), by Fondo Europeo de Desarrollo Regional (FEDER), and by the EU project eLIFT (Grant agreement 247868). 


\section{References}

1. B. Kundu, S. C. Kundu, Prog. Polym. Sci. 35, 1116-1127 (2010)

2. D. B. Weibel, W. R. DiLuzio, G. M. Whitesides, Nat. Rev. Microbiol. 5, 209-218 (2007)

3. E. A. Roth, T. Xu, M. Das, C. Gregory, J.J. Hickman, T. Boland, Biomater. 25, 3707-3715 (2004)

4. D. Soltman, V. Subramanian, Langmuir 24, 2224-2231 (2008)

5. C. C. Wu, D. N. Reinhoudt, C. Otto, V. Subramaniam, A. H. Velders, Small 7(8), 989-1002 (2011)

6. S. Lenhert, C. A. Mirkin, H. Fuchs, Scanning 32, 15-23 (2010)

7. D. B. Chrisey, A. Piqué, R. A. McGill, J. S. Horwitz, B. R. Ringeisen, D. M. Bubb, P. K. Wu, Chem. Rev. 103 (2), 553-576 (2003)

8. P. Serra, M. Colina, J. M. Fernández-Pradas, L. Sevilla, J. L. Morenza, Appl. Phys. Lett. 85, 1639-1641 (2004)

9. M. Colina, P. Serra, J. M. Fernández-Pradas, L. Sevilla, J. L. Morenza, Biosens. Bioelectron., 20, 1638-1642 (2005)

10. J. A. Barron, H. D. Young, D. D. Dlott, M. M. Darfler, D. B. Krizman, B. R. Ringeisen, Proteomics 5(16), 4138-4144 (2005)

11. I. Zergioti, A. Karaiskou, D. G. Papazoglou, C. Fotakis, M. Kapsetaki, D. Kafetzopoulos, Appl. Phys. Lett. 86, 163902, (2005)

12. V. Dinca, A. Ranella, M. Farsari, D. Kafetzopoulous, M. Dinescu, A. Popescu, C. Fotakis, Biomed. Microdevices 10, 719-725 (2008)

13. C. B. Arnold, P. Serra, A. Piqué, MRS Bulletin 32, 23-31 (2007)

14. A. Palla-Papavlu, I. Paraico, J. Shaw-Stewart, V. Dinca, T. Savopol, E. Kovacs, T. Lippert, A. Wokaun, M. Dinescu, Appl. Phys. A 102, 651-659 (2011)

15. A. Doraiswamy, R. J. Narayan, T. Lippert, L. Urech, A. Wokaun, M. Nagel, B. Hopp, M. Dinescu, R. Modi, R. C. Y. Auyeung, D. B. Chrisey, App. Surf. Sci. 252, 4743-4747 (2006)

16. N. T. Kattamis, P. E. Purnick, R. Weiss, C. B. Arnold, Appl. Phys. Lett. 91, 171120 (2007)

17. B. Hopp, T. Smausz, N. Kresz, N. Barna, Z. Bor, L. Kolozsvári, D. B. Chrisey, A. Szabó, A. Nógrádi, Tissue Engineering 11(11-12), 1817-1823 (2005)

18. J. M. Fernández-Pradas, M. Colina, P. Serra, J. Domınguez, J. L. Morenza, Thin Solid Films 27, 453-454 (2004) 
19. C. Unger, M. Gruene, L. Koch, J. Koch, B. N. Chichkov, Appl. Phys. A (2011) 103: 271277

20. M. Duocastella, J. M. Fernández-Pradas, P. Serra, J. L. Morenza, Appl. Phys. A 93, 453 (2008)

21. M. Duocastella, J. M. Fernández-Pradas, J. L. Morenza, P. Serra, J. Appl. Phys. 106, 084907 (2009)

22. M. Duocastella, J. M. Fernández-Pradas, J. L. Morenza, P. Serra, Thin Solid Films 518, 5321-5325 (2010)

23. A. I. Kuznetsov, C. Unger, J. Koch, B. N. Chichkov, Appl. Phys. A 106, 479-487 (2012)

24. H. Kim, R. C. Y. Auyeung, S. H. Lee, A. L. Huston, A. Pique, J. Phys. D: Appl. Phys. 43, $085101(2010)$

25. L. Rapp, J. Ailuno, A. P. Alloncle, P. Delaporte, Opt. Express 19(22), 21563-21574 (2011)

26. M. Duocastella, H. Kim, P. Serra, A. Piqué, Appl. Phys. A 106, 471-478 (2012)

27. M. Duocastella, M. Colina, J. M. Fernández-Pradas, P. Serra, J. L. Morenza Appl. Surf. Sci. 253, 7855-7859 (2007)

28. B. J. Kang, J. H. Oh, Thin Solid Films 518, 2890-2896 (2010)

29. H. Gau, S. Herminghaus, P. Lenz, R. Lipowsky, Science 283, $46-49$ (1999)

30. P. C. Duineveld, J. Fluid Mech. 477, 175-200 (2003)

31. J. Stringer, B. Derby, Langmuir 26 (12), 10365-10372 (2010)

32. J.-T. Wu, S. L.-C. Hsu, M.-H. Tsai, W.-S. Hwang, J. Phys. Chem. C 115, 10940-10945 (2011)

33. K. Y. Shin, S. H. Lee, J. H. Oh, J. Micromech. Microeng. 21, 045012 (2011)

34. A. M. J. van den Berg, A. W. M. de Laat, P. S. Smith, J. Perelaer, U. S. Schubert, J. Mater.

Chem. 17, 677-683 (2007) 


\section{Figure caption}

Figure 1 Optical microscopy image of a) printed droplets at different droplet spacings, $\Delta \mathrm{x}$ and b) $2 \mathrm{~mm}$ uniform line printed at $40 \mu \mathrm{m}$ droplet spacing; inset: area of the uniform line printed at $40 \mu \mathrm{m}$ droplet spacing. Printing direction is a) from top to bottom, and b) from left to right. Scale bar is $100 \mu \mathrm{m}$.

Figure 2 a) Time resolved images of liquid ejection and sessile droplet formation through LIFT at different delay times with respect to the laser pulse. The arrow indicates the point where the jet contacts the receiver substrate surface. b) Plot of the droplet diameter versus time corresponding to the liquid ejection and droplet formation displayed in (a). The uncertainty in the determination of the droplets diameter is of about 5\%. Figure adapted from Ref. 22.

Figure 3 Top views of the different instances of jet impingement depending on droplet spacing: a) $\Delta \mathrm{x}=120 \mu \mathrm{m}$, b) $\Delta \mathrm{x}=100 \mu \mathrm{m}$, c) $\Delta \mathrm{x}=80 \mu \mathrm{m}$, d) $\Delta \mathrm{x}=70 \mu \mathrm{m}$, e) $\Delta \mathrm{x}=60 \mu \mathrm{m}, \mathrm{f}) \Delta \mathrm{x}=50 \mu \mathrm{m}, \mathrm{g})$ $\Delta \mathrm{x}=40 \mu \mathrm{m}, \mathrm{h}) \Delta \mathrm{x}=30 \mu \mathrm{m}$ and i) $\Delta \mathrm{x}=20 \mu \mathrm{m}$. For each instance, the feature on the left represents the end of the line which is being printed, and on the right is sketched the perimenter (solid circle, final diameter $d_{0}$ ) that the next droplet would attain at the end of stage $S_{3}$, and the perimeter of the printed feature during stage $S_{2}$ (dashed circle, diameter $d_{1}$ ). All the sketches are to scale. 


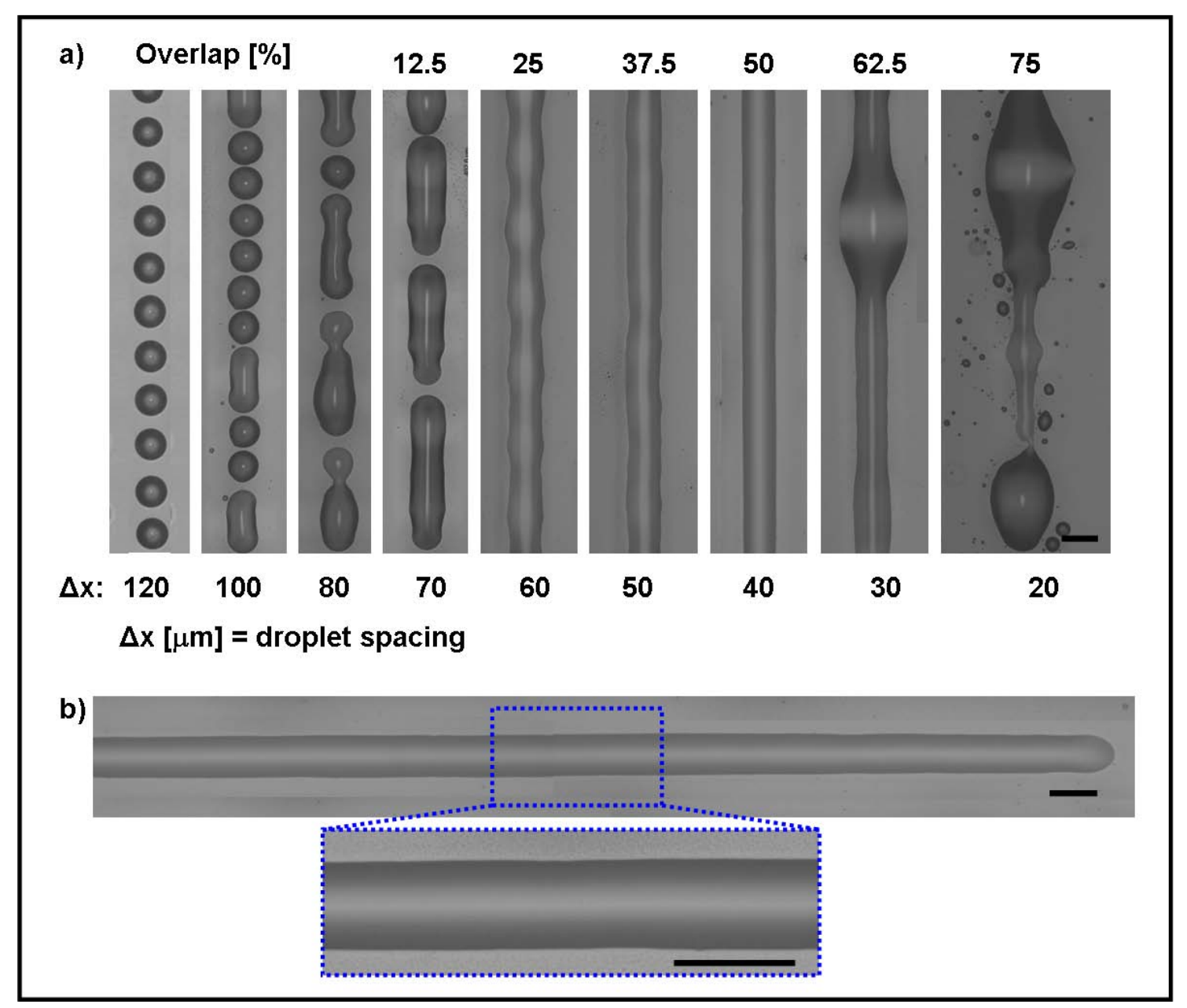

Figure 1 


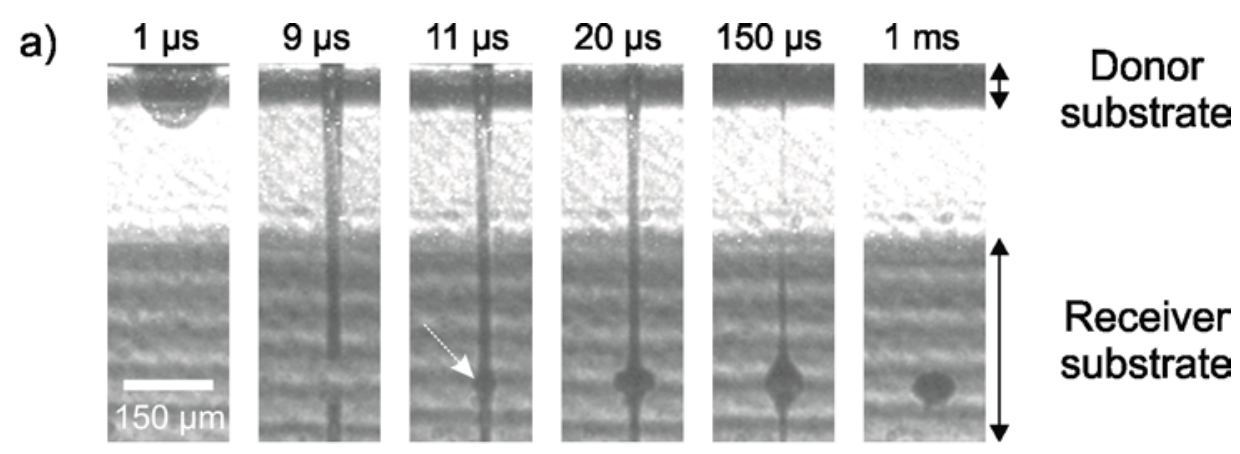

b)

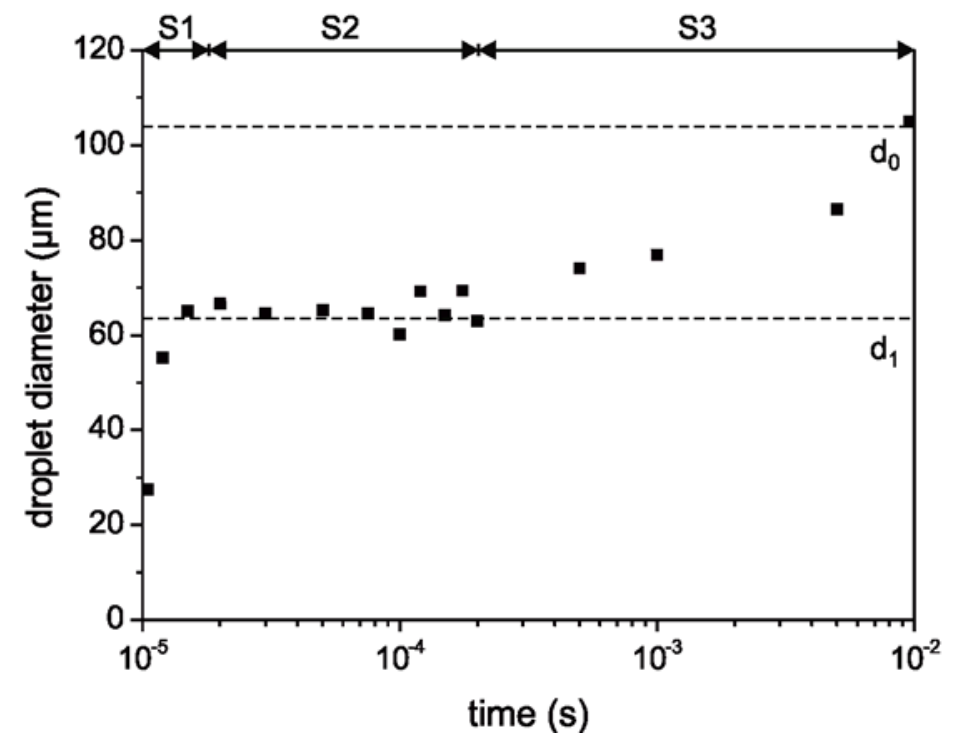

Figure 2 


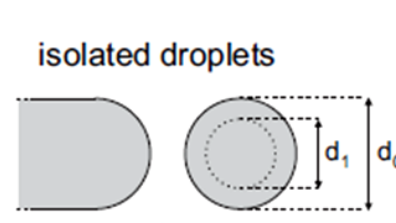

a) isolated droplets + pairs

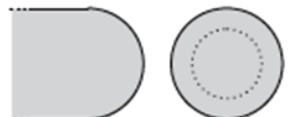

b)

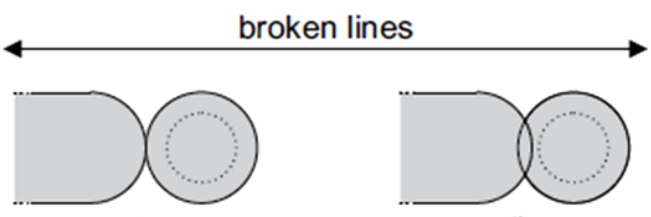

c) d)

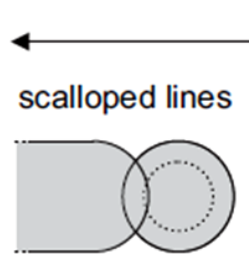

e)

continuous lines

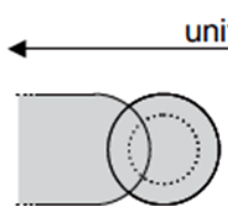

f)

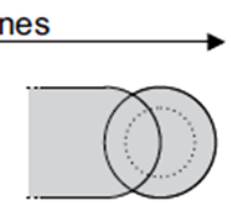

g)

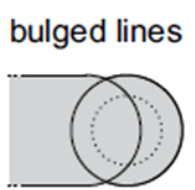

h)

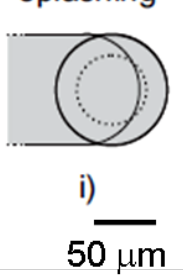

Figure 3 